University of Nebraska - Lincoln

DigitalCommons@University of Nebraska - Lincoln

20th \& 21st Century French and Francophone Modern Languages and Literatures, Department Studies International Colloquium

\title{
La dimension cinématographique du paysage dans l'œuvre littéraire de Julien Gracq
}

\author{
El Arbi El Bakkali \\ Université Abdelmalek Essaadi, Morocco
}

Follow this and additional works at: https://digitalcommons.unl.edu/ffsc2020

Part of the Comparative Literature Commons, French and Francophone Literature Commons, and the Other French and Francophone Language and Literature Commons

El Bakkali, El Arbi, "La dimension cinématographique du paysage dans l'œuvre littéraire de Julien Gracq" (2020). 20th \& 21st Century French and Francophone Studies International Colloquium. 20.

https://digitalcommons.unl.edu/ffsc2020/20

This Presentation is brought to you for free and open access by the Modern Languages and Literatures, Department of at DigitalCommons@University of Nebraska - Lincoln. It has been accepted for inclusion in 20th \& 21st Century French and Francophone Studies International Colloquium by an authorized administrator of DigitalCommons@University of Nebraska - Lincoln. 


\section{El Arbi El Bakkali}

Université Abdelmalek Essaadi, Morocco

\section{La dimension cinématographique du paysage dans l’ouvre littéraire de Julien Gracq}

Classé parmi les maîtres de la littérature contemporaine en France, Julien Gracq de son vrai nom Louis Poirier (professeur de géographie) a pu marquer sa carrière d'écrivain sans qu'il s'inscrive à aucun courant littéraire précis. Sa production littéraire est protéiforme et s'étale sur deux grandes périodes littéraires: la première (1938-1967) prenait du surréalisme et de la fiction comme cadre principal de création littéraire tandis que la seconde période (1967-1992) était axée sur des fragments d'autobiographie, réflexions sur la littérature et médiations géographiques. Il est important de souligner que le point commun entre ces deux périodes artistiques de l'auteur est l'omniprésence du paysage (fictif ou réel) où la lisière constitue chez Gracq un tremplin qui fait passer l'intrigue du récit d'un endroit clos (forêt ou château), à un autre ouvert (rivière ou mer). Selon Gracq, les personnages, les souvenirs (surtout d'enfance) et les mots eux-mêmes n'acquéraient un sens et une présence qu'en relation profonde avec la texture d'un sol c'est-à-dire la géographie du paysage. Ceci dit, dans ce travail nous avons essayé de mettre en exergue les aspects du paysage, qui se manifeste à travers l'œuvre de Gracq, dans le cadre cinématographique.

Ainsi, notre approche est une analyse littéraire qui accorde le plus grand intérêt à l'étude de l'espace ou plus précisément l'étude du paysage. A vrai dire, dans ce travail nous cherchons à mettre en exergue la dimension de l'espace gracquien en tant que composante littéraire dans le cinéma en prenant en considération bien entendu l'inacceptation explicite de Gracq pour l'adaptation cinématographique de ses œuvres. Pour Gracq, le cinéma est fort important que pour les œuvres simples tandis que pour une œuvre excellente, la création littéraire y compris l'évocation de l'espace dépasse largement l'art cinématographique.

20th \& 21st Century French and Francophone Studies International Colloquium, University of Nebraska-Lincoln, March 26-28, 2020.

https://digitalcommons.unl.edu/ffsc2020/

Copyright @ 2020 El Arbi El Bakkali

DOI: $10.32873 /$ unl.dc.ffsc.017 


\title{
A) Julien Gracq : un écrivain ayant le style d'un géographe.
}

Julien Gracq a dénoncé toute initiative de modélisation de la production littéraire parce que toute théorisation de l'écriture littéraire empiète sur le domaine des sciences exactes (dont les théories garantissent la progression et la fiabilité au niveau des résultats). Il considère ainsi la littérature comme une science dure en la privant de son aspect utopique, naturel, contemplatif et libre qui laisse le choix aux écrivains de produire sans prendre en considération le souci de suivre un certain nombre de recommandations et de consignes adoptées à l'unanimité par les groupes des littérateurs du XXème siècle. En effet, il chercha, dès ses débuts d'écrivain, à ne pas se soumettre aux théories de la mouvance littéraire de son époque, affirmant la liberté pour l'écrivain d'organiser ses idées naturellement sans avoir le souci de suivre un schéma déjà préparé. Selon Gracq, le fait d'écrire suivant un modèle précis vide la littérature de son essence définie dans le monde entier comme un exercice de production linguistique innovant et dépassant le simple message transmettant une idée ou traduisant une réalité. Lors d'un entretien, Gracq a bien développé sa propre manière d'écrire :

\begin{abstract}
J'avance en effet dans mon travail de phrase en phrase, de page en page en suivant la progression du récit. Je le suis comme un vecteur qui ne comporte pas de retour en amont, peut être parce que mes livres de fiction figurent tous plus ou moins la maturation d'un événement qui est presque d'ordre organique, et qui ne comporte pas de stase, ni de régression. C'est une démarche naturelle ${ }^{1}$.
\end{abstract}

Gracq fit preuve d'une grande originalité lorsqu'il choisit d'écrire sous forme de fragments pour se libérer définitivement de la notion de progression thématique qu'on rencontre dans la plupart des romans et récits. Quand on évoque la production littéraire de Gracq, il y a toujours un consensus qui se focalise sur l'esprit critique de l'auteur et son amour des paysages comme notion géographique par excellence. Ainsi, la production littéraire de Julien Gracq est-elle axée sur les trois sommets d'un triangle symbolisant sa démarche:

$>$ Evocations de paysages ;

$>$ Fragments autobiographiques ;

$>$ Réflexions sur la littérature.

La notion de paysage est l'un des mots clés de l'œuvre de Gracq, sans doute parce qu'elle découle de sa formation de géographe. Pour celui-ci, le paysage est d'une importance capitale car il joue un rôle au même titre que les personnages d'un roman. Les paysages sont présents dans toute sa production littéraire, mais il n'est pas sans importance de remarquer que les types de paysages varient avec les différentes périodes au cours desquelles il écrit. Dans la première période, où Julien Gracq fait appel à la fiction, il crée (ce terme de créer ne lui plairait pas!) $)^{2}$ des paysages fictifs qui ne se réfèrent en rien à des paysages réels, mêmes s'ils apparaissent par ailleurs comme des «synthèses » (c'est un terme qu'il employa) très concevables. La seconde période, marquée par un aspect fragmentaire de la production

\footnotetext{
${ }^{1}$ Julien Gracq, Entretiens, Paris : Editions José Corti, 2002, p. 300.

${ }^{2}$ Julien Gracq disait dans Entretiens à la page 167 : «Je n'aime pas l'emploi du mot 'création' en littérature, et encore moins en critique. Il n'y a création qu'à partir de rien, et la littérature n'est au mieux que recomposition, réassemblage, de sensations, de perceptions et de souvenirs ».
} 
littéraire se manifeste par des descriptions réelles de lieux précis, le réalisme de l'auteur s'exprimant ainsi par une certaine volonté de coller au réel. Mais cette volonté de coller au réel (ou de lire et écrire le réel) le conduit à transformer ce réel en particulier en se désolidarisant des contraintes traditionnelles de l'espace et du temps. La formation de géographe de l'auteur fusionne avec son talent de poète pour donner un réel imaginaire, esthétique, fascinant parce que conduisant à un univers particulier où l'auteur semble se fondre lui-même avec le monde qu'il décrit par des mots savants ou non. Il affirme cela lors d'un entretien avec Jean-Louis Tissier en 1978 :

Quand j'emploie un terme géographique (cela m'arrive), c'est un mode d'excuse ; cela veut dire: "voilà, je n'ai pas de meilleur mot que le mot savant pour exprimer ce que je veux dire, je m'en excuse ». L'italique a beaucoup d'autres sens. Mais pour les termes géographiques, pour moi, c'est souvent celui-làa ${ }^{3}$

En effet, le paysage, une des composantes de la géographie physique que Julien Gracq pratiqua jusqu'à sa retraite, prend une grande place dans l'univers littéraire de l'auteur et particulièrement dans la seconde partie de sa production; il le dit clairement en répondant à une question de Jean-Louis Tissier lors de l'entretien précité :

En fait, au début, j'ai écrit surtout des romans. Les paysages concrets, les choses vues y tiennent moins de place. Dans la dernière partie, j'ai écrit les deux volumes de Lettrines, Les Eaux étroites qui renvoient à des lieux précis. Il y a donc davantage de réalisme, alors que tous les paysages des romans sont des paysages remaniés ou synthétiques mais je crois que mon goût pour les paysages $n$ 'a pas changé de nature ${ }^{4}$.

Outre le paysage, l'esprit critique des œuvres littéraires occupe aussi une place constante dans la totalité de l'œuvre de Gracq qui était loin d'être en harmonie littéraire avec ses contemporains souvent liés à l'existentialisme (Sartre, Camus). Les romans existentialistes se déroulent la plupart du temps dans le monde de la ville, dans l'atmosphère des conversations de café, des discussions, des contacts dans la rue ; la ville est en quelque sorte la plate-forme de ce type d'écriture. Gracq réfute ce mode d'écriture dans ses critiques, et cherche d'autres lieux, fictifs, en dehors de la ville. La vision géographique de Gracq d'un paysage se concrétise immédiatement par un sentiment, une caresse ou une odeur. Quant aux rares personnages du récit gracquien, ils sont capables de tisser des relations très profondes avec les paysages. En vérité, comme les données géologiques et météorologiques recèlent leur vérité, le paysage, dans l'œuvre romanesque aussi, se manifeste toujours dans sa présence véritablement active, il lance des appels et interroge : point de site, généralement désert, qui n'ait de signification. Point de lieu vacant qui ne constitue, à lui seul, un événement. Point de territoire qui ne contienne ses hiéroglyphes. Il se peut même que les paysages demandent à être déchiffrés comme des cryptogrammes. Aller à la rencontre du secret, du texte sensible

\footnotetext{
3 J. Gracq, Entretiens, op.cit.p.34.

${ }^{4}$ J. Gracq, Entretiens, op.cit.p.45.
} 
de l'univers qui appelle un décryptage, et accueillir ce qu'il devient dans sa singularité : cette volonté de compréhension rejoint la quête surréaliste d'une totalité sans fissure où la conscience pénètre librement les choses, et s'y baigne sans cesser d'être, où l'irréversibilité du temps s'abolit avec le passé et le futur.

\section{B) Dimension LiTTÉRAIRE DU PAYSAGE CHEZ GRACQ :}

Pourquoi écrit-on?

On n'est pas écrivain sans avoir le sentiment que le son, dans le mot, vient lester le sens, et que le poids dont il est ainsi doté peut l'entraîner légitimement, à l'occasion de singulières excursions centrifuges. L'écriture comme la lecture est mouvement, et le mot s'y comporte en conséquence comme un mobile dont la masse, si peu qu'elle se réduise, ne peut jamais être tenue pour nulle et peut sensiblement infléchir la direction. ${ }^{5}$

Une question se pose et même s'impose : comment la notion de mouvement est-elle saisie par Julien Gracq? Julien Gracq est connu à cause son admiration pour la lisière comme lieu d'inspiration et de production littéraire. Ce lieu présente un paysage sombre où l'on se situe à la limite d'un autre coin inconnu et étranger ; cela provoque chez les personnages de l'œuvre de Gracq un état de peur, de curiosité, d'attente, d'extase, d'aventure et de rêve. Toutes ces images constructrices de ce paysage mystérieux et frontalier épousent essentiellement les couleurs : blanc et noir. Ainsi, ces couleurs qui symbolisent le vide, le néant et le silence sont les couleurs les plus fréquentes dans l'œuvre gracquienne puisque leurs fréquences ${ }^{6}$ sont : 600 occurrences pour la couleur noire et 384 occurrences pour la couleur blanche. Il est vrai que le noir est opposé au blanc comme sa contre couleur mais, chez Gracq, elles contribuent toutes les deux à l'incarnation des traits symboliques qui constituent le paysage inspiratoire de la frontière à travers son œuvre littéraire. Dans cette optique, Jacqueline Michel $^{7}$ écrit que la lisière se profile dans le texte gracquien comme un silence matérialisé qui, tel celui des eaux étroites « un doigt sur les lèvres, debout et immobile » impose l'écoute des signes qui dénoncent la puissance de «l'en-face », de l'extrême limite. Cette lisière pourrait être une forêt, un rivage, une mer ou généralement une ligne que Jacqueline Michel commente comme suit :

Ligne où se joignent fini et infini, commencement et fini, le rivage rend manifeste le mystère de la limite. Particulièrement dans la presqu'île, il apparaît comme une ligne de silence où s'épuise le réel et naît le rêve : "brusquement ici la vie changeait de clé » le rivage fait pressentir les possibilités d'une révélation de

\footnotetext{
5 J. Gracq, En lisant en écrivant, op.cit.p.148.

${ }^{6}$ EL Arbi EL Bakkali, «Etude de statistique lexicale : quelques aspects ambigus des couleurs dans l'œuvre littéraire de Julien Gracq » in Ambiguïtés, Actes du colloque d'Albi, Langages et signification/ 2012.

${ }^{7}$ Jacqueline Michel, Une mise en récit du silence, Le Clézio /Bosco/Gracq, Paris, José Corti, 1989, p.78.
} 
l'en-face, de l'ailleurs saisi comme une réserve du nouveau. Et le héros gracquien se tient sur le bord de cette ouverture, le regard fixe sur les lointains de silence ${ }^{8}$.

En effet, la lisière pour Gracq est l'endroit le plus intense, le plus propice, c'est la serrure qui ouvre les portes de l'attente vers un sixième sens ambigu. Lors d'un entretien Julien Gracq dévoilait le vrai rôle de la lisière dans ses écrits :

Confins, lisières, frontières, effectivement, sont des lieux qui m'attirent en imagination: ce sont des lieux sous tension, et peut-être cette tension est-ellematérialisée, localisée- l'équivalent de ce qu'est la tension latente entre ses personnages pour un romancier psychologue : un stimulant imaginatif initial. Il arrive le plus souvent que les personnages, dans mes romans, soient eux-mêmes mis, par rapport à la société, dans une situation de "lisière », par une guerre, par des vacances, par une mise en disponibilité quelconque ${ }^{9}$.

À vrai dire la lisière, incarnant un climat d'attente, n'attribue aucune présence aux personnages chez Julien Gracq. Pour cela, l'écriture gracquienne s'inscrit aussi dans un autre cadre méthodologique de l'écocritique qui se donne comme objectif principal d'analyser le rapport entre la littérature et l'environnement. Malgré sa grande diversité de méthodologies et de théories, l'écocritique se distingue d'autres approches littéraires de par son insistance sur le rôle du monde non-humain dans les textes littéraires, artistiques, cinématographiques, enfin, dans tout texte compris au sens large du terme.

Selon Michel Collet, le paysage est un enjeu stratégique. Il n'est pas seulement un terrain d'action ni un objet d'étude : il donne à penser, et à penser autrement. Il nous propose, entre autres choses, un modèle pour l'invention d'un nouveau type de rationalité qu'il appelle la "pensée-paysage". La juxtaposition des deux termes permet de suggérer à la fois que le paysage donne à penser, et que la pensée se déploie comme paysage.

Cette "pensée-paysage" qui émane aujourd'hui des œuvres, mais aussi des pratiques paysagères, qui récusent les méfaits du modernisme et réinventent sous des formes et avec des moyens nouveaux l'ancienne alliance entre l'être humain et son environnement. A vrai dire cette combinaison se manifeste chez Julien Gracq comme la coexistence entre le corps et la terre. Autrement dit, la littérature de Gracq le géographe entretient des connexions spéciales entre le corps (les sens) et la terre (les paysages) .

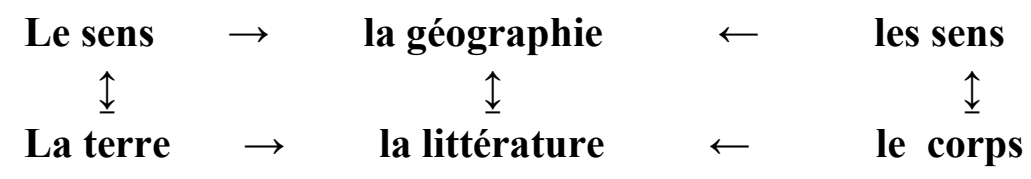

De fait, l'œuvre de Julien Gracq se distingue par une attention constante portée aux paysages et par une volonté toujours plus grande d'établir une communication entre les forces vives du

\footnotetext{
${ }^{8} \mathrm{~J}$. Michel, Une mise en récit du silence, Le Clésio/Bosco/Gracq, op. cit, p.79.

9 J. Gracq, Entretiens, op.cit.p.171.
} 
cosmos et l'âme individuelle. En somme, la formation géographique de Julien Gracq (le paysage et la lisière) était derrière cette coexistence, sinon cette intimité, entre le sens linguistique et les sens.

\section{C) Dimension cinématographique du paysage gracquien :}

Dès les débuts du cinéma, transposer des œuvres littéraires à l'écran parait discutable voire polémique dans la mesure où comment le cinéma pourrait-il traduire un langage aussi différent de lui que celui de la littérature sans le dénaturer? Jamais l'adaptation cinématographique ne saurait égaler la saveur d'une œuvre originale. La pratique de l'adaptation est immédiatement soupçonnée de bloquer l'imaginaire du lecteur et d'en figer la représentation mentale en "donnant vie » aux personnages romanesques. Ainsi, on trouve chez Julien Gracq la conviction que le film limite le rêve par «somme quasi infinie d'informations instantanées que nous livre l'image alors que la parcimonie, la pauvreté même des notations de la phrase romanesque correspondante ouvrent à l'imagination le champ infini des possibles.

Selon Gracq, Le cinéma échoue le plus souvent lorsqu'il entreprend de transcrire selon ses moyens les très grands romans, ceux où la langue est utilisée selon sa capacité d'évocation, non d'information. Ici les images qu'il impose sans possibilité de choix font trop souvenir du pouvoir fâcheusement réducteur- pour l'imagination- qui est le sien par rapport à la fiction écrite. En revanche, il a magnifié nombre de romans de gare, écrits en pur style d'information, grâce à la présence inégalable de l'image : cela fait équilibre.

A vrai dire, L’image ne suggère pas, n'évoque pas : elle est avec une force de présence que le texte écrit n'a jamais, mais une présence exclusive de tout ce qui n'est pas elle. L'image plastique refoule et exclut toutes les autres. En effet la notion d'image comme source de sens nous impose d'incarner celle du paysage et du langage. Le cinéma ${ }^{10}$ prétend se doter d'un langage à lui, d'un langage qui échappe au langage. Un langage dépend intrinsèquement de l'image, du son et de la lumière. Ces trois composantes créaient des structures visuelles et des mouvements conçus comme les seuls conducteurs.

Deux langages différents se manifestent : le langage des images ne reflète pas le langage des mots. Par exemple, les ellipses au cinéma (flash-back, fondus) représentent le temps qu'on ne voit pas. Ainsi, il y a un décalage réel entre une scène racontée et une séquence de film. La voix-off au cinéma fait entendre au spectateur les pensées du personnage; Le procédé est beaucoup plus fréquent dans les romans avec le point de vue omniscient. Un film de 2 heures ne peut pas reprendre tous les événements et les gestes d'un récit de 300 pages. Le cinéaste doit donc faire des choix. Il peut décider de supprimer certains détails, de

\footnotetext{
${ }^{10}$ Gracq disait durant un entretien: « Il faut reconnaitre d'ailleurs qu'il y a toujours eu dans le rapport du public avec le cinéma un élément de consommation régulière et périodique où les hauts et les bats de la qualité se marquent moins que dans les autres arts. Le langage le souligne ; on dit qu'on « va au cinéma », on ne dit pas qu'on « va à la librairie » ou à la bibliothèque : on va acheter tel ou tel livre »
} 
réduire le nombre de personnages, de passer des faits sous silence. Enfin, en adaptant, le cinéaste propose sa lecture, sa vision de l'œuvre, son interprétation.

En effet, évoquer le langage cinématographique c'est soumettre les textes littéraires à l'épreuve de la langue parlée, avec ses hésitations, ses intermittences, ses silences, ses répétitions. Les dialogues n'ont pas simplement vocation à faire passer un message mais à exprimer un aspect du comportement. Ceci dit, l'adaptation de l'œuvre gracquienne au cinéma pourrait être effectuée sans problème? Sachons que Gracq défend souvent le cheminement naturel quant à la création de l'œuvre littéraire par contre le cinéma est tout à fait artificiel : c'est une industrie de l'image. Sommes nous devant une vraie incompatibilité cinéma/ littérature.

Puisque le cinéma se base sur l'image comme vecteur directeur du sens et selon les préférences du paysage chez Gracq comme pièce capitale de création littéraire. Pouvons nous parler d'une équivalence image/ paysage. L'adaptation cinématographique de l'œuvre gracquienne se manifeste surtout dans le roman Un balcon en forêt. Dans cette optique, Julien Gracq disait lors d'un entretien :

«Cela m'a beaucoup frappé quand Un balcon en forêt a été adapté; la dramatique m'a plu d'ailleurs, mais c'était autre chose : par exemple, dans le roman, il y a quatre soldats qu'on ne voit pas, dont on sait seulement qu'ils sont là; à l'écran, on les voit, ce qui est tout à fait différent. Pour qu'un grand roman devienne un très bon film (il n'y a pas d'impossibilité absolue), il faut que le film soit autre chose, il s'agit de chercher une sorte d'équivalent qui ne se limite pas à la transposition visuelle ».

Il est clair que Julien Gracq a évoqué le cinéma souvent dans ses critiques ou pendant ses entretiens avec différents journalistes. Selon lui, le roman ne doit jamais faire voir, il est luimême vision. On ne peut pas dessiner ni inventer une scène de roman; et lui-même, lorsque il écrit, il ne voit pas ses personnages, ce n'est pas ainsi que cela se passe: c'est la sonorité du mot, de la phrase, qui évoque des présences, mais un peu nébuleuses. Tout se passe comme si le romancier, ou le lecteur de romans, disposait de plusieurs écrans : ils voient les choses de face, mais parallèlement, l'auteur ayant la possibilité de retourner en arrière, d'utiliser les divers temps du verbe, le présent cohabitant avec le futur et le passé, il existe aussi des écrans latéraux où ils perçoivent d'autres éléments. Tandis qu'au cinéma, le flash-back est un procédé brutal, qui consiste à plaquer un morceau de passé...

Le roman gracquien nous informe davantage sur les raisons qui poussent les êtres à agir, leurs motivations, leur passé; l'image elle, fixe des moments d'émotion mais ne permet pas aussi facilement la superposition des plan temporels à moins d'utiliser des techniques particulières comme la surimpression ou le flash-back qui plongent le spectateur dans les souvenirs des personnages.

Pour clore, au cinéma, Est-ce que le paysage Gracquien a besoin d'un texte ? Puisque Gracq choisit le paysage naturel qui se parle. Le cinéma muet pourrait-il bien interpréter les textes de Gracq ? 


\section{Références bibliographiques :}

Alain, Michel, Paysages et mémoires /des eaux étroites à Un balcon en forêt, Editions Cécile défaut, 2007.

Amossy, Ruth, Parcours symboliques chez Julien Gracq : Le Rivage des Syrtes, Paris, Sedes, 1982.

Bachelard, Gaston, La Poétique de l'espace, PUF ,1958.

Butor, Michel, Essais sur le roman. Gallimard, 1992.

Canovas, Fréderic, L'écriture rêvée, Paris, l'Harmattan, 2000.

Collot, Michel, Paysage et Poésie, Paris, José Corti ,2005.

Collot, Michel, La Pensée-Paysage Philosophie, arts, littérature, Broché, 2011.

EL Bakkali, EL Arbi, Etude de statistique lexicale : quelques aspects ambigus des couleurs dans l'œuvre littéraire de Julien Gracq, dans " Ambiguïtés », Actes du XXXIII colloque d'Albi, Langages et Signification, Le Mirail, Toulouse, (2012), p. 253-264.

Genette, Gérard, Espace et langage, dans «Figures I », Seuil, Collection Points, (1966), p. $\sim 101-108$.

Genette, Gérard, La littérature et l'espace, dans «Figures II», Seuil, Collection Points, (1969), p. 43-48.

Gracq, Julien , Un beau ténébreux, Paris, José Corti,1945.

Gracq, Julien , Liberté grande, Paris, José Corti, 1946.

Gracq, Julien , Le roi pêcheur, Paris, José Corti,1948.

Gracq, Julien , André Breton, aspects d'un écrivain, Paris, José Corti,1948.

Gracq, Julien , La littérature à l'estomac, Paris, José Corti,1950.

Gracq, Julien , , Le rivage des Syrtes, Paris, José Corti,1951.

Gracq, Julien , Penthésilée, Paris, José Corti,1954.

Gracq, Julien , Un balcon en forêt, Paris, José Corti,1958.

Gracq, Julien , Préférences, Paris, José Corti,1961.

Gracq, Julien , Lettrines 1, Paris, José Corti,1967.

Gracq, Julien , La presqu'île, Paris, José Corti,1970.

Gracq, Julien , Lettrine 2, Paris, José Corti,1974.

Gracq, Julien , Les eaux étroites, Paris, José Corti,1976.

Gracq, Julien , En lisant en écrivant, Paris, José Corti,1980.

Gracq, Julien , Autour des sept collines, Paris, José Corti,1988. 
Gracq, Julien , Carnets du grand chemin, Paris, José Corti,1992.

Gracq, Julien , Entretiens,, Paris, José Corti,2002.

Grossman, Simone, Julien Gracq et le surréalisme, Paris, José Corti, 1980.

Haddad, Hubert, Julien Gracq. La forme d'une vie, Paris, Editions Zulma, 2004.

Jean-Louis, L'écrivain au travail , dans « Le journal », 16 mai 1970.

Michel, Jacqueline, Une Mise en récit du silence, Le Clézio-Bosco-Gracq, Paris, José Corti, 1986.

Murat Michel, L'enchanteur réticent, Essai sur Julien Gracq, Paris, José Corti, 2004.

Posthumus, Stéphanie, Écocritique et "ecocriticism". Repenser le personnage écologique, dans «La pensée écologique et l'espace littéraire », Cahier Figura : le Centre de recherche sur le texte et l'imaginaire, (2014), vol. 36, p. 15-33.

Westphal, Bertrand, La Géocritique. Réel, fiction, espace, Editions de minuit, 2007.

"Gracq cinéphobe », a paru dans «Les dernières confidences de Julien Gracq » (Nouvel Observateur, $\mathrm{n}^{\circ} 2254,17-23$ janvier 2008, p.98-101). Des propos recueillis par Noël Herpe et Michka Assayas le 16 décembre 1995, extrayons l'avis de Julien Gracq sur les adaptations cinématographiques des romans (p.101; version longue en ligne). 